\title{
Ending the "Neglect" to End Neglected Tropical Diseases
}

\author{
Pin Yang ; Xiao-nong Zhou ${ }^{1,2, *}$
}

January 30, 2022 will mark the third World Neglected Tropical Diseases (NTD) Day. The slogan for this year World NTD Day is "A New Day in the fight against NTDs" (https://worldntdday.org/). While human immunodeficiency virus (HIV)/acquired immune deficiency syndrome (AIDS), tuberculosis, and malaria were regarded as the three major infectious diseases and have received greater treatment and research funding, NTDs have almost disappeared in the global health policy agenda to gain recognition in 2015 with the Sustainable Development Goals (SDG target 3.3).

NTDs are a diverse group of tropical infections threatening more than 1.7 billion people living in the most marginal communities of the world. Worldwide, an estimated 200,000 deaths and 19 million disabilityadjusted life years are annually attributed to NTDs. Every year, NTDs cause billions of US dollars loss in direct health costs, indirect costs of lost productivity, and socioeconomic attainment. In January 2021, the World Health Organization (WHO) launched the second 2021-2030 NTD roadmap to identify critical gaps and the actions required to reach the targets set for 2030. Discussing how to implement the NTDs control program in line with the new road map represented in "Ending the neglect to attain the Sustainable Development Goals: a road map for neglected tropical diseases 2021-2030" (1), is a research priority. In order to achieve the targets, there is urgent need for collaborative actions to combat NTDs through cooperation in the control activities, coherence of various resources, and coordination of different control programs in developing regions of Africa, Asia, and the Americas. The most important action would be to end the "neglect" to achieve the goal of ending the epidemic of NTDs under the framework of the One Health approach (2).

\section{END THE "NEGLECT" BY BRINGING THE STAKEHOLDERS TOGETHER}

NTDs are caused by a variety of pathogens, such as viruses, bacteria, protozoa, and parasitic worms. According to the global report on NTDs by the WHO, NTDs include 20 kinds of infectious diseases, caused by pathogens of viruses $(n=3)$, bacteria $(n=4)$, protozoa $(n=4)$, helminths $(n=8)$, fungi $(n=2)$, ectoparasites $(n=1)$, as well as one noncommunicable disease (3).

Due to different routes of transmission, the WHO recommends following an integrated control strategy for national NTDs control program. The control strategy to interrupt the transmission of NTDs relied on the following integrated approaches: preventive chemotherapy and vector control reducing the exposure risk, disease management ensuring early diagnosis and treatment, quality patient care in mental health and/or surgery and/or rehabilitation, and crosscutting by the One Health approaches including water, sanitation, and hygiene. Through implementation of these integrated interventions, the NTDs burden causing poverty, disability, and stigmatization of affected population would be alleviated, especially in resource-limited areas.

To efficiently implement and sustain those integrated interventions, the World NTD Day is an appropriate opportunity for the following: 1) bringing civil society advocates, community leaders, global health experts, and policymakers together to discuss NTD control actions; 2) bringing all stakeholders working across the diverse NTD landscape together to unify partners behind the common goal of ending the epidemic of NTDs; and 3) bringing all resources together to reduce the burden of patients with NTDs and further decline inequity, such as loss of education, loss of productivity, and lack of access to healthcare.

\section{END THE EPIDEMIC OF NTDS BY APPLYING THE ONE HEALTH APPROACH}

In 2019, 3 international organizations including the WHO, Food and Agriculture Organization of the United Nations (FAO), and World Organization for 
Animal Health (OIE) published "Taking a Multisectoral, One Health Approach: A Tripartite Guide to Addressing Zoonotic Diseases in Countries" (4). This document is a symbol that One Health will be a powerful weapon to fight infectious diseases of poverty, since One Health is a collaborative, multidisciplinary, and multisectoral approach that can address urgent, ongoing, or potential health threats at the humananimal-environment interface (ecosystem) at all levels, e.g., local, subnational, national, regional, and global levels.

The One Health approach claims to be the best choice for the NTDs control program because of the following reasons. First, NTDs are caused by multiple species of pathogens with a series of complexities, including complexity in routes of transmission, complexity in integration of intervention, and complexity in evaluation of the control program, etc. To solve such complexities in one framework, the One Health approach is able to uncover the original courses and provide solutions to those complexities. Second, the systems thinking approach has been exercised in the formulation of the new road map for beating NTDs by the WHO in 2020. Through the gap analysis for each NTD presented by heat map, the WHO has proposed three innovative ways of designing the intervention strategy to beat NTDs, i.e., accelerating programmatic actions, intensifying crosscutting approaches, and changing operational models and culture to facilitate country ownership, based on the new road map for combating NTDs issued in 'Ending the neglect to attain the Sustainable Development Goals: a road map for neglected tropical diseases 2021-2030.' Third, the benefits of applying the One Health approach to the NTDs control program can certainly achieve optimal outcomes such as the following: 1) to accelerate technical progress with more effective interventions targeting multiple NTDs in one approach; 2) to effectively implement the NTDs program systematically from strategy to service delivery and from implementation to evaluation; 3) to mobilize multisectoral action and collaboration for resources mobilization; 4) to integrate anti-NTDs activities on common health delivery platforms to make possible the targeting of a series of diseases together; 5) to integrate NTD control within national health systems under the framework of universal health coverage; and 6) to integrate with other sectors to improve the governance ability of the NTDs program.

\section{ACHIEVEMENTS OF \\ SCHISTOSOMIASIS CONTROL IN CHINA, A MODEL FOR \\ IMPLEMENTING THE ONE HEALTH APPROACH}

Schistosomiasis is transmitted by freshwater snails and has been listed as a neglected tropical disease by the WHO. The life cycle of schistosomes is complex, involving humans, various mammals, aquatic snails, and a variety of freshwater bodies. More than 200 million people are still suffering from schistosomiasis worldwide.

Schistosomiasis japonica is caused by Schistosoma japonicum and distributed in China, the Philippines, and Indonesia. It is a zoonosis with more than 40 species of mammals involved in transmission. Schistosomiasis is distributed in 12 provincial-level administrative divisions (PLADs) and has affected hundreds of millions of people in China. Great achievements of the national schistosomiasis control program have been gained with the help of the One Health concept, such as multisectoral cooperation, various regional coordination from local to provincial and national level communities, and disciplinary integration (5). The major lessons in the process of the One Health approach that improved the schistosomiasis control program are summarized as follows.

First, the government took leadership supported by multisectoral cooperation to improve the governance capacities. The national schistosomiasis control program of China launched in the 1950s, was initiated by a government leading group established under the leadership of the Chairman Mao Zedong. Then, the call for schistosomiasis elimination in all applicable areas by Chairman Mao Zedong became the final goal of the national schistosomiasis program in China. With the effort of generations of professionals from institutions/universities and residents from communities, remarkable achievements have been obtained in China. For example, all 450 endemic counties have reached the criteria of transmission control (prevalence $<1 \%$ in humans and domestic animals) in 2015, and 435 out of 450 (96.7\%) endemic counties have achieved the criteria of transmission interruption as of 2020 (6). These achievements were attributed to multisectoral 
cooperation, such as modification of snail habitats along the canals by agriculture irrigation engineering projects or water conservation projects, and control or reduction of snail densities in the forest or marshland through forest engineering projects or planting projects, which were performed by multiple sectors at the county, provincial, and national levels ( 7 ). Based upon the progress of schistosomiasis control program, the State Council launched in late 2014 the national schistosomiasis elimination program, with its goal to interrupt disease transmission by 2025 and totally eliminate schistosomiasis by 2030 . Up to now, the governance mechanism with Inspection in Spring and Meeting in Autumn is still executed annually to ensure the national schistosomiasis control and elimination program moves forward to eradication by 2030; that is to say that schistosomiasis control and elimination efforts have never been neglected over seven decades.

Second, various regional coordination from local community to provincial and national levels improved efficiency of the program. The involvement of local communities in the national schistosomiasis control and elimination program ensures that all activities can be promptly performed at the community level, with coordination of the county CDCs. To ensure the quality of the activities, monitoring and evaluation have to be implemented by professional staff from county- or prefecture-level CDCs. The surveillance and response systems have been well established through coordination by the National Institute of Parasitic Diseases of China CDC, which formed the surveillance network covering all endemic counties, including passive surveillance network covering a total of 850 surveillance villages, an active surveillance network, and a sentinel surveillance network (8). In addition, two cross-region cooperative networks have been established. One is the cross-lake regional cooperative network on schistosomiasis control which involved five provinces, including Jiangsu, Anhui, Jiangxi, Hunan, and Hubei. The other one is the cooperative network for PLADs surveillance, with all counties achieving elimination of schistosomiasis in 8 PLADs, including Guangdong, Guangxi, Fujian, Zhejiang, and Shanghai. Those two networks contribute to schistosomiasis elimination cooperatively by information exchanges and resource sharing, improving the efficacy of schistosomiasis elimination program by targeting mobile population, blocking snail spreading, etc. All of the aforementioned cooperative activities have been well implemented through coordination from the central government to local communities.

Third, disciplinary integration improved innovation from control to elimination. All the disease control programs could be further improved once innovative approaches are employed. The national schistosomiasis control program of China was integrated with implementation research since 1992 when the World Bank Loan Project on Schistosomiasis Control in China was launched. This has not only promoted the capacity to execute the program but also integrate the disciplinary knowledge or technology to accelerate the progress of the program. By applying knowledge from epidemiology, population genetics, modern biology, mathematic modelling, etc., an integrated strategy on schistosomiasis control with an emphasis of infectious source control has been developed and implemented since 2006 , which greatly promotes the schistosomiasis control program marching from control toward elimination. Therefore, such an integrated strategy with infectious source control provides references to design the national schistosomiasis elimination program in order to achieve the final goal of schistosomiasis elimination in China by 2030. In addition, a lot of innovative research and development products, including fast diagnostics, drug candidates and their use in clinics, and new molluscicide approaches, facilitated by good governance mechanisms with multisectoral cooperation have been made possible and feasible, so that outcomes of the schistosomiasis elimination program have been accomplished with a higher cost-effectiveness (9). It is clearly illustrated that the positive bi-interaction between the program execution and implementation research could be significantly improved when disciplinary integration is performed. Therefore, the long-term impact to the schistosomiasis elimination program will certainly achieve the final goal of being schistosomiasis-free in China.

\section{TAKING THE ONE HEALTH APPROACH TO ACHIEVE THE TARGETS FOR 2030}

Substantial progress has been made in past years 43 countries have eliminated at least one NTD, and 600 million people no longer require treatment for NTDs. However, many of the targets set for 2020 were not achieved. For the next decade, much work still needs to be done as at least 1.74 billion people require interventions against NTDs. Looking to the future 
years, the new WHO NTD roadmap set global targets and cross-cutting targets aligned with both the WHO's Thirteenth General Programme of Work, 2019-2023, and the SDGs. To achieve the targets, further multisectoral actions should be taken to beat all 20 diseases and disease groups through the application of the One Health approach. Chinese experience in eliminating some NTDs, in conjunction with poverty reduction, multisectoral cooperation, and disciplinary integration, can serve as a model for other countries or regions (7).

Conflicts of interest: Xiao-nong Zhou is an editorial board member of the journal China CDC Weekly. He was not involved in the peer-review or handling of the manuscript. The authors have no other competing interests to disclose.

Funding: National Key Research and Development Program of China (No. 2021YFC2300800, 2021YFC2300804).

\section{doi: $10.46234 / \mathrm{ccdcw} 2022.018$}

\# Corresponding author: Xiao-nong Zhou, zhouxn1@chinacdc.cn.

\begin{abstract}
${ }^{1}$ National Institute of Parasitic Diseases, Chinese Center for Disease Control and Prevention; Chinese Center for Tropical Diseases Research; WHO Collaborating Centre for Tropical Diseases; National Center for International Research on Tropical Diseases, Ministry of Science and Technology; Key Laboratory of Parasite and Vector Biology, Ministry of Health, Shanghai, China; ${ }^{2}$ School of Global Health, Chinese Center for Tropical Diseases Research, Shanghai Jiao Tong University School of Medicine, Shanghai, China.
\end{abstract}

Submitted: December 20, 2021; Accepted: January 28, 2022

\section{REFERENCES}

1. World Health Organization. Ending the neglect to attain the sustainable development goals: a road map for neglected tropical diseases 2021-2030. Geneva: World Health Organization. 2020. https://apps. who.int/iris/handle/10665/338565. [2021-12-2].

2. World Health Organization. Tripartite and UNEP support OHHLEP's definition of "One Health". 2021. https://www.who.int/news/item/01 12-2021-tripartite-and-unep-support-ohhlep-s-definition-of-one-health. [2021-12-2].

3. World Health Organization. Neglected tropical diseases. 2021. https:// www.who.int/health-topics/neglected-tropical-diseases\#tab=tab_1. [2021-12-2].

4. World Health Organization (WHO), Food and Agriculture Organization of the United Nations (FAO), World Organisation for Animal Health (OIE). Taking a multisectoral, one health approach: a tripartite guide to addressing zoonotic diseases in countries. Geneva: World Health Organization. 2019. https://www.oie.int/fileadmin/ Home/eng/Media_Center/docs/EN_TripartiteZoonosesGuide_ webversion.pdf. [2021-12-2].

5. Monnier N, Barth-Jaeggi T, Knopp S, Steinmann P. Core components, concepts and strategies for parasitic and vector-borne disease elimination with a focus on schistosomiasis: a landscape analysis. PLoS Negl Trop Dis 2020;14(10):e0008837. http://dx.doi.org/10.1371/journal.pntd. 0008837.

6. Zhang LJ, Xu ZM, Yang F, Dang H, Li YL, Lü S, et al. Endemic status of schistosomiasis in People's Republic of China in 2020. Zhongguo Xue Xi Chong Bing Fang Zhi Za Zhi 2021;33(3):225-33. (In Chinese).

7. Engels D, Zhou XN. Neglected tropical diseases: an effective global response to local poverty-related disease priorities. Infect Dis Poverty 2020;9(1):10. http://dx.doi.org/10.1186/s40249-020-0630-9.

8. Xu J, Li SZ, Zhang LJ, Bergquist R, Dang H, Wang Q, et al. Surveillance-based evidence: elimination of schistosomiasis as a public health problem in the Peoples' Republic of China. Infect Dis Poverty 2020;9(1):63. http://dx.doi.org/10.1186/s40249-020-00676-5.

9. Liang $\mathrm{S}$, Abe EM, Zhou XN. Integrating ecological approaches to interrupt schistosomiasis transmission: opportunities and challenges. Infect Dis Poverty 2018;7(1):124. http://dx.doi.org/10.1186/s40249 018-0506-4. 